Few-Body Systems Suppl. 0, 1-?? (2017)

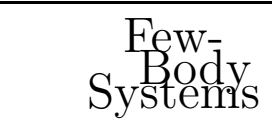

(C) by Springer-Verlag 2017 Printed in Austria

\title{
Front-form calculation of the deuteron EM form factors
}

\author{
J. Carbonell* 1, V.A. Karmanov ${ }^{\dagger 2}$ \\ ${ }^{1}$ Institut des Sciences Nucléaires, 53 av. des Martyrs, 38026 Grenoble, France \\ ${ }^{2}$ Lebedev Physical Institute, Leninsky Prospekt 53, 117924 Moscow, Russia
}

\begin{abstract}
The deuteron form factors are calculated in the framework of the relativistic nucleon-meson dynamics. The relativistic effects change considerably the S- and D-waves of the deuteron, result in the dominating extra component in the deuteron wave function and generate the contact interaction between the nucleon, mesons and photon. The prediction for the polarization observable $t_{20}$ is in good agreement with the recent data of TJNAF/CEBAF.
\end{abstract}

\section{Introduction}

We calculate the deuteron form factors in the framework of the explicitly covariant version of light-front dynamics (see for review [1]). In this approach one can use in full measure the knowledge of the nonrelativistic wave functions and incorporate selfconsistently the relativistic effects. We assume that the nucleons interact with each other by exchanging the same mesons that are incorporated in the $N N$ potential, however we don't use any nonrelativistic potential approximation.

\section{Wave function}

In the explicitly covariant formulation of light-front dynamics the wave function is defined in the space-time on the plane formally given by the light-front equation $\omega \cdot x=0$, where $\omega$ is the four-vector with $\omega^{2}=0$. This restores the relativistic covariance in comparison to the standard light-front approach. The latter is obtained as a particular case for $\omega=(1,0,0,-1)$. The wave functions depend on the orientation of the light front, as they should. This dependence is covariantly parametrized in terms of the four-vector $\omega$.

The explicit covariance allows to construct the general form of the lightfront wave function for a system with given spin. The relativistic deuteron

*e-mail: carbonel@isn.in2p3.fr

†e-mail: karmanov@sci.lebedev.ru 
wave function on the light front contains six spin components, in contrast to two components - S and D-waves - in the nonrelativistic case. Its general form was found in [3]. In our calculation we keep only three dominating components $f_{1}, f_{2}$ and $f_{5}$.

The relativistic deuteron wave function was calculated in [7 by a perturbative way incorporating exact relativistic OBEP kernel found in light-front dynamics, and the nonrelativistic wave function as the starting point. For the kernel the set of six mesons $(\pi, \eta, \rho, \omega, \delta$ and $\sigma)$ and the parameters corresponding to the Bonn model [5] were used.

In nonrelativistic region of $k$ the components $f_{1}$ and $f_{2}$ found by this way turn into the usual S- and D-waves, whereas other components become negligible. However, starting from $k \approx 0.5 \mathrm{GeV} / \mathrm{c}$ the component $f_{5}$ dominates over all other components, including $f_{1}$ and $f_{2}$.

The physical meaning of this dominating extra component has been clarified [6] by comparing, in $1 / m$ approximation, the analytical expression for the amplitude of the deuteron electrodisintegration near threshold with the nonrelativistic one, including meson exchange currents. For the isovector transition, in the region, where so called pair term with the pion exchange dominates, this component (together with similar component in the scattering state) automatically incorporates $50 \%$ of the contribution of the pair term and therefore dominates too. Another $50 \%$ is given by the contact (instantaneous) interaction (see below).

\section{Electromagnetic vertex}

A peculiarity of the light-front dynamics is the presence of the so called instantaneous (or contact) interaction $N N B \gamma$, where $B$ is the meson $\pi, \rho$, etc. This is a trace of disappeared diagrams corresponding to vacuum fluctuations. The fermion fields in this interaction are sandwiched with the factor $\hat{\omega}=\omega_{\mu} \gamma^{\mu}$ that in the standard approach is the well known $\gamma^{+}$(in addition to the standard factors of the nucleon-meson Lagrangian, like $\gamma_{5}, \gamma_{\mu}$, etc.). Therefore the full electromagnetic vertex which we use to calculate the deuteron form factors corresponds to the sum of the impulse approximation and of the contact interaction. It partially takes into account the many-body currents. For the contact interaction we take into account the sum over all six mesons contributing to the Bonn potential, with the parameters of the Bonn model [5].

\section{Finding form factors}

The next step is to extract the deuteron form factors from the electromagnetic vertex. The physical amplitudes should not depend on the orientation of the light-front plane. However in practice, because of the incompatibility between the transformation properties of the approximate e.m. current and the wave function, the nonphysical $\omega$ dependence survives in the on-energy shell deuteron electromagnetic vertex. Due to covariance, the general form of this dependence 
can be found explicitly and then separated from the physical form factors. The explicit formulas extracting the physical form factors $\mathcal{F}_{1}, \mathcal{F}_{2}, \mathcal{G}_{1}$ from the nonphysical contributions were found in refs. [7].

The polarization observable $t_{20}$ measured in experiment is a combination of the form factors (see e.g. [1]). Besides momentum transfer it depends also explicitly on $\tan ^{2} \frac{1}{2} \theta$.

\section{$5 \quad$ Numerical results and conclusions}

Our prediction [1], 2] for the polarization observable $t_{20}$ together with the most recent measurements are shown in figure 1 . The calculation is in close agreement with the existing experimental data in all the momentum range, including the new results obtained at TJNAF/CEBAF [8] which corresponds to $\theta=70^{\circ}$.

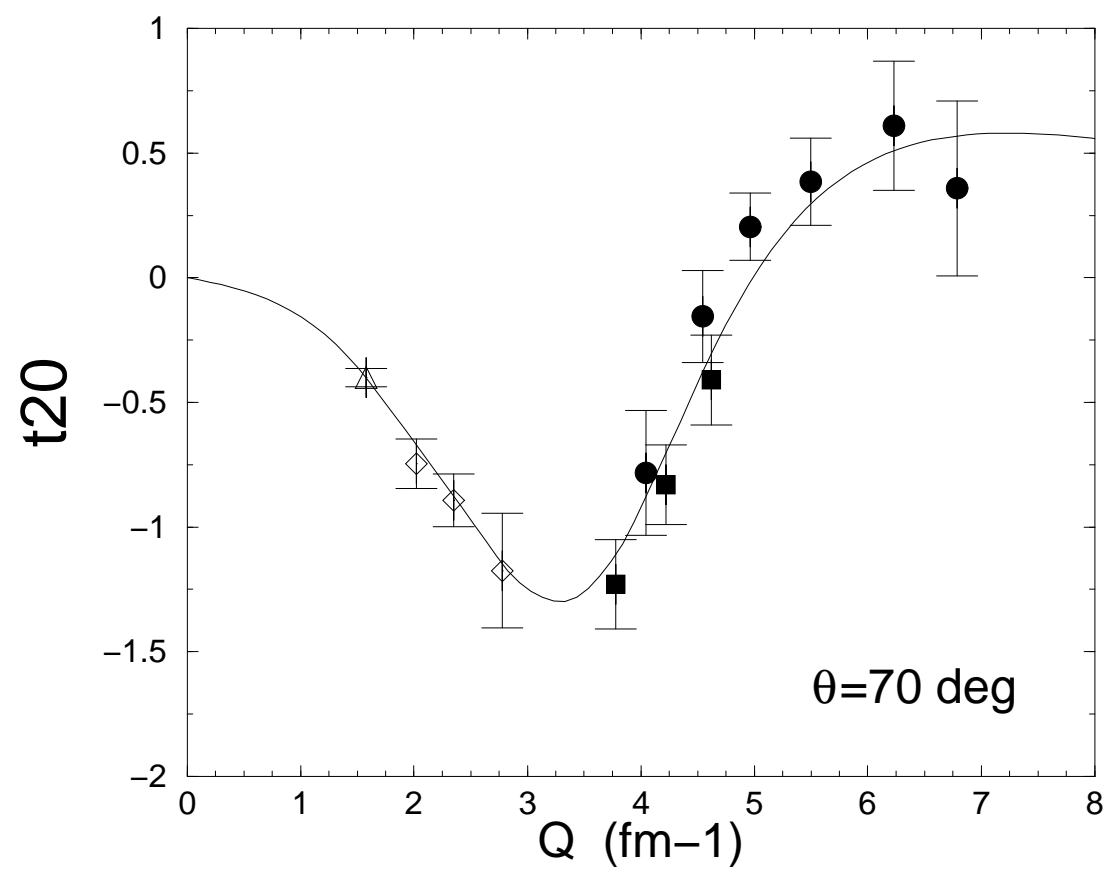

Figure 1. The deuteron tensor polarization $t_{20}$ at $\theta=70^{\circ}$ compared to most recent experimental data: triangle from [9], diamonds from [10], squares from [11], circles from 8

This coincidence means that the deuteron structure at small distances is understood rather well. It is given by the relativistic nucleon-meson dynamics. In its turn, the relativistic nucleon-meson dynamics is well described theoretically, 
in the framework of the phenomenological nucleon-meson Lagrangian. The parameters of this Lagrangian are found phenomenologically. Their calculation in the framework of QCD is a separate problem.

This allows us to make the general conclusion that: in a large extent the relativistic nuclear physics can be developed independently of its derivation from $Q C D$.

However, these results can be improved in many respects. An exact solution of the equation for the deuteron is indeed feasible. It implies the redefinition of the parameters of the NN kernel in the framework of the light-front equations. This necessitates also a careful treatment of higher order contributions to the kernel. Finally, the calculation of electromagnetic observables should include higher Fock states ( $N N \pi$, etc.) and the meson exchange currents corresponding to the direct interaction of the photon with the intermediate mesons.

\section{Acknowledgement}

The authors are sincerely grateful to B. Desplanques and J.-F. Mathiot for many useful discussions, valuable remarks and helpful advices.

\section{References}

1. J. Carbonell, B. Desplanques, V.A. Karmanov and J.-F. Mathiot; Physics Reports, 300 (1998) 215

2. J. Carbonell and V.A. Karmanov: Preprint ISN-98.54, to be published

3. V.A. Karmanov: Nucl. Phys. A362 (1981) 331

4. J. Carbonell and V.A. Karmanov: Nucl. Phys. A581 (1995) 625

5. R. Machleidt, K. Holinde and Ch. Elster: Phys. Reports, 149 (1987) 1

6. B. Desplanques, V.A. Karmanov and J.-F. Mathiot: Nucl. Phys. A589 (1995) 697

7. V.A. Karmanov and A.V. Smirnov: Nucl. Phys. A546 (1992) 691; A575 (1994) 520

8. S. Kox et al.: European Conf. on El.-Mag. Int. with Nucleons and Nuclei, Santorini (Greece), October 1997;

E.J. Beise et al: 16th European Conf. on Few-Body Problems in Physics, Autrans (France), June 1998

9. M. Ferro-Luzzi et al: Phys. Rev. Lett. 77 (1996) 2630

10. M. Ferro-Luzzi et al: Nucl. Phys. A631 (1998) 190

11. I. The et al: Phys. Rev. Lett. 67 (1991) 163 